Edith Cowan University

Research Online

ECU Publications Pre. 2011

2001

The duration derby : a comparison of duration based strategies in asset liability management

Harry Zheng

David E. Allen

Lyn C. Thomas

Follow this and additional works at: https://ro.ecu.edu.au/ecuworks

Part of the Statistics and Probability Commons

Zheng, H., Allen, D., \& Thomas, L. (2001) The duration derby : a comparison of duration based strategies in asset liability management. Perth, Australia: Edith Cowan University.

This Other is posted at Research Online.

https://ro.ecu.edu.au/ecuworks/7111 


\title{
The Duration Derby: A Comparison of Duration Based Strategies in
}

\section{Asset Liability Management}

\author{
By \\ Harry Zheng \\ Lyn C, Thomas \\ University of Southampton \\ and \\ David E. Allen \\ Edith Cowan University \\ June 2001 \\ Working Paper 01.09 \\ ISSN: 1323-9244
}

School of Finance and Business Economics Working Paper Series

Corresponding Author and Address:

Professor David E. Allen

School of Finance and Business Economics

Faculty of Business and Public Management

Edith Cowan University

Joondalup Campus

Joondalup WA 6027

Phone: $61+894005471$

Fax: $\quad 61+894005271$

email: d.allen@ecu.edu.au 


\begin{abstract}
Macaulay duration matched strategy is a key tool in bond portfolio immunization. It is well known that if term structures are not flat or changes are not parallel, then Macaulay duration matched portfolio can not guarantee adequate immunization. In this paper the approximate duration is proposed to measure the bond price sensitivity to changes of interest rates of nonflat term structures. Its performance in immunization is compared with those of Macaulay, partial and key rate durations using the US Treasury STRIPS and Bond data. Approximate duration turns out to be a possible contender in asset liability management: it does not assume any particular structures or patterns of changes of interest rates, it does not need short selling of bonds, and it is easy to set up and rebalance the optimal portfolio with linear programming.
\end{abstract}




\section{Introduction}

Duration is a useful way of making a rough assessment of the effect of interest rate changes on single bonds and portfolios of bonds. (See Bierwag [1987], Bierwag, Corrado, and Kaufman [1990].) If one only could use two numbers to describe the characteristics of a bond the obvious ones are its price and its duration. Duration has also proved effective in matching asset portfolios and liability portfolios by matching their durations, though recent developments in decomposition and sampling aspects of stochastic programming means that this more precise approach is becoming more viable for realistic problems. (See Birge and Louveaux [1998].)

However there are difficulties with the original Macaulay duration approach. It requires that the yield curve for the bond is flat even though the gilt market is usually suggesting something different and it does not deal with default risk explicitly. This paper reviews the first of these issues. An extension of the Macaulay duration, partial duration (Cooper [1977]) has been suggested as a way of dealing with non-flat yield curves. In this paper the idea of an approximate duration is introduced which is closer to the Macaulay duration idea of a second number to describe the relationship of an asset, liability or portfolio of such to interest rates. Unlike the Macaulay duration though this can be thought of as the median of the cash flow of the bond rather than the mean and hence cannot be obtained for a portfolio of bonds directly from the durations of the individual bonds. However a linear programming method of calculating this duration measure is described in the paper in the case of asset liability management.

The effectiveness of these duration measures is investigated by describing a simulation experiment using US Treasury STRIPS and Bond data to see how well these duration measures choose a portfolio of assets to match a given cash flow of liabilities. Five duration measures are compared in this experiment. The first is the Macaulay duration. Two are partial durations, - 
one applied to a given form of the yield curve and the other based on a key rate model. The other durations are both versions of the approximate duration idea.

Section two reviews the Macaulay duration and discusses the partial and key rate durations for non-flat term structures. Section three introduces the approximate duration approach. Section four describes how duration matching strategies can be applied to asset liability management problems. Section five deals with the "horse race"-the derby-between the five asset management strategies based on the different definitions of duration. It describes the way the experiment is performed and discusses the results.

\section{Macaulay and Partial Durations}

The Macaulay duration of a bond can be identified with the maturity of a zero-coupon risk free bond which has the same value and the same response to a small change in interest rates as the original bond. Thus if a bond has an income stream $c(t), t=1, \ldots, T$, over separate periods until its maturity at $T$, and $r$ is the implied interest rate or yield to maturity of the bond, the value of the bond $V$ satisfies

$$
V=\sum_{t} \frac{c(t)}{(1+r)^{t}}
$$

If one matches this by a zero-coupon bond which pays out $R$ at time $D$ so that its value is $V_{0}=R /(1+r)^{D}$ and both bonds have the same response to small changes of interest rates, then one would require

$$
V=V_{0} \text { and } \frac{d V}{d r}=\frac{d V_{0}}{d r}
$$

This leads to the standard definition of Macaulay duration for a risk-free bond, namely,

$$
D=\frac{1}{V} \sum_{t} \frac{t c(t)}{(1+r)^{t}} .
$$


The definition of Macaulay duration is based on the idea that the term structure is flat and that the only changes are parallel shifts. This is not what the market assumes and this has led to other definitions of duration. Suppose the term structure is not flat and the risk free spot rates are given by a vector $\mathbf{r}=\left(r_{1}, r_{2}, \ldots, r_{T}\right)$ then the value of a bond with income stream $c(t)$ is

$$
V(\mathbf{r})=\sum_{t} \frac{c(t)}{\left(1+r_{t}\right)^{t}}
$$

If, on the other hand, the risk-free forward rates are given by a vector $\mathbf{f}=\left(f_{1}, f_{2}, \ldots, f_{T}\right)$ then the value of a bond with income stream $c(t)$ is

$$
V(\mathbf{f})=\sum_{t} \frac{c(t)}{\prod_{s \leq t}\left(1+f_{s}\right)}
$$

Whichever formulation is used, one has to model the term structure or equivalently the discount factor $b(t)$ where

$$
V(b)=\sum_{t} b(t) c(t)
$$

and $b(t)=\left(1+r_{t}\right)^{-t}$ in $(3)$ and $b(t)=1 / \prod_{s \leq t}\left(1+f_{s}\right)$ in (4). There are two main approaches to modeling the term structure. The first is to choose a specific form of the yield curve and use the market data to estimate its parameters. Thus Haugen [1997] suggested a spot rate curve of the form

$$
r(t)=(a+b t) e^{-d t}+c .
$$

The parameters can be easily estimated using nonlinear regression methods and the model has the advantage that the parameters have an obvious interpretation: $a=r(0)-r(\infty)$ is the difference of the short rate and the long rate, $b=r^{\prime}(0)+d(r(0)-r(\infty))$ is related to the short rate slope and the overall structure of interest rates, $c=r(\infty)$ is the long rate, and $d=-r^{\prime \prime}(\infty) / r^{\prime}(\infty)$ is the ratio of curvature to slope in the long run but is also the rate of 
convergence to the long rate.

A second approach is to describe the movements in the term structure by a set of factors. In this case it is assumed that

$$
r(t)=\sum_{i} a_{i} F_{i}(t)+w(t)
$$

where $w(t)$ is a stochastic process with zero mean. The factors $F_{i}(t)$ are determined empirically (see Dahl [1993]) using factor analysis on the historical returns of pure discount bonds or the historical estimated term structures. Ho [1992] suggested that changes of spot rate curves are determined by changes of some key rates. Suppose for example the first, fifth and twenty-fifth year spot rates are taken as key rates, and changes of them are $a_{1}, a_{2}, a_{3}$, respectively. Then spot rate curves can be defined as

$$
r(t)=r_{0}(t)+\left\{\begin{array}{l}
\frac{1}{4}\left((5-t) a_{1}+(t-1) a_{2}\right), \quad t \leq 5 \\
\frac{1}{20}\left((25-t) a_{2}+(t-5) a_{3}\right), \quad 5 \leq t \leq 25 \\
a_{3}, \quad t \geq 25
\end{array}\right.
$$

where $r_{0}$ is the initial spot rate curve. In both cases one ends up with a discount function $b(t, \mathbf{a})$ which is a function of a few critical parameters, i.e. $b(t, \mathbf{a})=b\left(t, a_{1}, a_{2}, \ldots, a_{n}\right)$. Thus whichever model of spot rate (zero coupon bond yield) curve one chooses, one arrives at a model for the value of a bond which depends on a vector of parameters $\mathbf{a}=\left(a_{1}, a_{2}, \ldots, a_{n}\right)$ which describe the spot rates or forward rates, so that

$$
V(\mathbf{a})=\sum_{t} b(t, \mathbf{a}) c(t)
$$

Following the analogy with the derivation of the Macaulay duration, one would ask what is the maturity of a zero coupon risk-free bond paying out $R$ at time $D$ (so its value is $V_{0}(\mathbf{a})=R b(D, \mathbf{a})$ ) that has the same value as the previous bond and the same response to small changes in risk-free rates. 
The problem is that there are now a number of ways the risk free rate can change, not just the parallel shifts in the term structure that is implicit in the Macaulay duration. What is normally suggested in the literature is to calculate the duration for each of the ways that this rate can change and seek to match asset and liability portfolios in each of these durations. One assumes that each change in the risk free rate corresponds to a change in one of the parameters that make up the risk free interest rate term structure and hence the discount factors $b(t, \mathbf{a})$. Cooper [1977] first suggested this approach and subsequently these durations became called partial durations. Given the bond price model of (7), then the $i$ th partial duration is

$$
D_{i}=-\frac{1}{V(\mathbf{a})} \frac{\partial V(\mathbf{a})}{\partial a_{i}}
$$

As examples, consider using the spot rate curve formulation of (5) and assume the short rate, the short rate slope, and the long rate are independent factors. This leads to partial durations of the form

$$
\begin{aligned}
& D_{1}=\sum_{t} t(1+d t) e^{-d t} C(t) \\
& D_{2}=\sum_{t} t^{2} e^{-d t} C(t) \\
& D_{3}=\sum_{t} t\left(1-(1+d t) e^{-d t}\right) C(t)
\end{aligned}
$$

where $C(t)=c(t)(1+r(t))^{-(t+1)} / V(\mathbf{a})$. Here $D_{1}$ is the duration to the short rate, $D_{2}$ to the short rate slope, $D_{3}$ to the long rate.

If key rates are used to describe term structure model, then their partial durations, or key rate durations, can be computed in the same way. For example, consider using the first, fifth, and twenty-fifth year rates as key rates as in (6), then partial durations of bonds to these key 
rates are

$$
\begin{aligned}
& D_{1}=\sum_{t \leq 5} \frac{1}{4}(5-t) t C(t) \\
& D_{2}=\sum_{t \leq 5} \frac{1}{4}(t-1) t C(t)+\sum_{5<t \leq 25} \frac{1}{20}(25-t) t C(t) \\
& D_{3}=\sum_{5<t \leq 25} \frac{1}{20}(t-5) t C(t)+\sum_{t>25} t C(t)
\end{aligned}
$$

where $C(t)=c(t)\left(1+r_{0}(t)\right)^{-(t+1)} / V(\mathbf{a})$. Here $D_{1}$ is the duration to the first year key rate, $D_{2}$ to the fifth year key rate, and $D_{3}$ the twenty-fifth year key rate.

Given one is seeking to allow for all the possible changes in the term structure that one has identified one would expect fitting portfolios by matching all their partial durations would be much more successful than just matching on the one Macaulay duration. This is what Chambers, Carleton, and McEnally [1988] investigated and they did find an improvement in immunizing the terminal values of the portfolio, when transaction costs are ignored.

\section{Approximate Durations}

There is an alternative duration measure that may be more robust than the Macaulay duration and which has the advantage that the user can specify which types of change to the interest rate term structure are of most concern to him. This approach minimizes the weighted sum of the errors of the sensitivity of the bond to changes in each of the parameters in the yield curve. This duration, called the approximate duration, is obtained as follows for a bond whose price $V(\mathbf{a})$ is given by (7). As in the case with the Macaulay duration, one wishes to find the maturity, $D$, of the zero-coupon risk free bond, paying $R$ which most closely matches the weighted sum of the individual changes in the yield curve. The price of such a bond is $V_{0}(\mathbf{a})=R b(D, \mathbf{a})$. The weighting put on the individual changes is given by the weights vector $w$. So the approximate 
duration is defined by finding the $D(w)$ where

$$
V(\mathbf{a})=V_{0}(\mathbf{a}) \text { and } \sum_{i} w_{i}\left|\frac{\partial V(\mathbf{a})}{\partial a_{i}}-\frac{\partial V_{0}(\mathbf{a})}{\partial a_{i}}\right| \text { is minimized }
$$

The approximate duration of a bond is a generalization of Macaulay duration to non-flat term structures of interest rates, since if the term structure is flat with interest rate $r$ as the only factor, then $V(r)=\sum_{t} c(t) /(1+r)^{t}$ and $V_{0}(r)=R /(1+r)^{D}$, and (11) reduces to $V(r)=V_{0}(r)$ and $w_{1}\left|d V / d r-d V_{0} / d r\right|$ is minimized. The minimum is achieved if and only if $D=\sum_{t} t c(t)(1+$ $r)^{-t} / V(r)$ which is the Macaulay duration. One can define the approximate duration for any types of interest rate models described above. As an example its calculation is described in the case where the parameters are all the forward interest rates, f. In this case $D(w)$ is the value where as in (11)

$$
V(\mathbf{f})=V_{0}(\mathbf{f}) \text { and } \sum_{s=1}^{T} w(s)\left|\frac{\partial V(\mathbf{f})}{\partial f_{s}}-\frac{\partial V_{0}(\mathbf{f})}{\partial f_{s}}\right| \text { is minimized. }
$$

Since bonds usually have an integer valued maturity one should first look at the duration $D(w)$ which is the integer that minimizes this value. This is the nearest integer approximate duration. The difference between the $s$ th partial derivatives in (12) if $s \leq D$, is

$$
\frac{\partial V}{\partial f_{s}}-\frac{\partial V_{0}}{\partial f_{s}}=-\sum_{t \geq s} \frac{b(t) c(t)}{\left(1+f_{s}\right)}+\frac{V_{0}(\mathbf{f})}{\left(1+f_{s}\right)}=\sum_{t<s} \frac{b(t) c(t)}{\left(1+f_{s}\right)}
$$

where $b(t)=1 / \prod_{u \leq t}\left(1+f_{u}\right)$ is a discount factor. The last equality comes from the fact that $V_{0}(\mathbf{f})=V(\mathbf{f})$. If $s>D$ then

$$
\frac{\partial V}{\partial f_{s}}-\frac{\partial V_{0}}{\partial f_{s}}=-\sum_{t \geq s} \frac{b(t) c(t)}{\left(1+f_{s}\right)}
$$

If cash flows $c(t)$ are non-negative for all $t$, then the function to be minimized in (12) can be written as

$$
E(D)=\sum_{s \leq D} \tilde{w}(s) \sum_{t<s} b(t) c(t)+\sum_{s>D} \tilde{w}(s) \sum_{t \geq s} b(t) c(t)
$$


where $\tilde{w}(s)=w(s) /\left(1+f_{s}\right)$. Therefore the approximate duration $D(w)$ is the minimum solution of $E(D)$ over $D=1,2, \ldots, T$, i.e.,

$$
E(D(w))=\min _{1 \leq D \leq T} E(D)
$$

If some cash flows $c(t)$ are negative (such as in the case where bonds might be short sold), then a linear integer programming can be used to find the approximate duration as follows:

$$
\text { Minimize } \sum_{s} \tilde{w}(s)(\alpha(s)+\beta(s))
$$

subject to the following constraints, for all $s=1, \ldots, T$

$$
\sum_{t \geq s} b(t) c(t)+\alpha(s)-\beta(s)=V(\mathbf{f})\left(\sum_{u=1}^{s} y(u)\right)
$$

and

$$
\sum_{u=1}^{T} y(u)=1
$$

and

$$
\alpha(s), \beta(s), y(s) \geq 0
$$

for all $s=1, \ldots, T$ and $y(s)$ are integers.

$D(w)$ is a different measure of stability than the Macaulay duration family of measures which concentrate on one dimensional changes in the forward rates. To see this compute the difference $E(D+1)$ and $E(D)$ in the positive cash flow case to get

$$
E(D+1)-E(D)=\tilde{w}(D+1)\left(\sum_{t<D+1} b(t) c(t)-\sum_{t \geq D+1} b(t) c(t)\right)
$$

and it follows that the minimum occurs at

$$
D(w)=\max \left\{D: \sum_{t \leq D} b(t) c(t)<\sum_{t \geq D+1} b(t) c(t)\right\}
$$


This is the median of the discounted cash flows whereas the Macaulay duration is the mean. Note that this result holds no matter what the weighting $w$ of the importance of the different periods sensitivities, provided they are non-zero. So if one assumes the forward rates are able to move independently rather than together in a one dimensional family, medians of the cash flow may be more appropriate than the means as measures of duration.

Unlike the mean, the median of a linear combination of measures need not be the linear combination of the individual medians and hence the value $D(w)$ of a portfolio of bonds has to be calculated by considering the total cash flow from the portfolio rather than by combining the durations of the individual bonds.

\section{Asset Liability Management with Transaction Costs}

Asset liability management is concerned with selecting a bond portfolio such that value of asset is the same as that of liability no matter how interest rates change. Suppose there are $n$ bonds in an asset portfolio. Then the value of the asset is

$$
V_{A}=V_{1} x_{1}+\cdots+V_{n} x_{n}
$$

where $V_{j}$ is the present value of bond $j$ and $x_{j}$ is the number of bond $j$ in the portfolio. If the term structure is flat and has only parallel shifts, then the duration of the asset portfolio is derived from those of individual bonds by

$$
D_{A}=D_{1} y_{1}+\cdots+D_{n} y_{n}
$$

where $D_{j}$ is the Macaulay duration of bond $j$ and $y_{j}=x_{j} V_{j} / V_{A}$ is the current price weighted proportion of bond $j$ in the portfolio. An immunized asset liability portfolio can be set up with 
two equality constraints:

$$
V_{A}=V_{L} \text { and } D_{A}=D_{L}
$$

where $V_{L}$ is the present value of the liability and $D_{L}$ the Macaulay duration of the liability. There may be several solutions to the two constraints. An objective function is then used to select an "optimal" solution that minimizes the cost, or maximizes the yield, etc.

Suppose there are transaction costs of buying or selling bonds, which are proportional to number of bonds bought or sold then minimizing the transaction cost of each period is a natural choice of objective function. If the portfolio is periodically rebalanced with the policy that all old bonds are sold or bought at rebalancing time and a new portfolio is set up to hedge again the new liability, the transaction cost is $\left|x_{1}\right|+\cdots+\left|x_{n}\right|$, the total number of bonds bought and sold.

In the previous two sections, several variants of duration are introduced. Each of them can be used as a way of managing an asset portfolio which is meant to cover a liability by matching the asset durations with the liability durations. The following strategies are considered, where initially assume no short selling of bonds in the asset portfolio is allowed, i.e., $x_{j} \geq 0$ for all $j$.

Macaulay duration matched strategy. The first approach is to use the Macaulay durations of bond portfolios for non-flat term structures. Suppose $D_{j}$ is Macaulay duration for bond $j$, $j=1, \ldots, n$. Then the Macaulay duration $D_{A}$ of the portfolio is defined by (16). This approach is using the yield of each bond to define its duration, rather than the yield of the portfolio, see Bierwag, Corrado, and Kaufman [1990] for a discussion of this point. The latter approach is more difficult here because the durations are themselves needed to define what is the optimal portfolio. The optimal portfolio is selected by solving a linear programming problem that minimizes the total number of bonds $\sum_{j} x_{j}$ in the portfolio subject to two equality constraints $V_{A}=V_{L}$ and 
$D_{A}=D_{L}$

Nearest Integer Approximate duration matched strategy. The second approach is to use approximate duration. Since no short selling of bonds is allowed, all cash flows are non-negative. The approximate duration of the bond portfolio $D_{A}$ is computed from

$$
E_{A}\left(D_{A}\right)=\min _{D} E_{A}(D)
$$

where $E_{A}(D)$ is defined by (13) with cash flow $\sum c_{j}(t) x_{j}$ at time $t$. Exchange order of summation to get

$$
E_{A}(D)=\sum_{i} E_{j}(D) x_{j}
$$

where $E_{j}(D)$ is defied by (13) with cash flow $c_{j}(t)$ at time $t$. Note that one can not calculate the approximate duration this way if short selling is allowed since some cash flows are then negative and $E_{A}(D)$ is not defined by (13), instead a linear programme problem must be solved. To have approximate duration matched portfolio one requires $D_{A}=D_{L}$ which implies $D_{L}$ is the minimum solution to (18), which is equivalent to the following inequality constraints:

$$
\sum_{j} E_{j}\left(D_{L}\right) x_{j} \leq \sum_{j} E_{j}(D) x_{j}
$$

for $D=1, \ldots, T$. The optimal portfolio is selected by solving a linear programming problem that minimizes $\sum_{j} x_{j}$ subject to one equality constraint $V_{A}=V_{L}$ and a set of inequality constraints (19).

Approximate duration matched strategy. In the above approach one simply requires the portfolio to satisfy a number of inequality constraints, there will be a number of portfolios which satisfy all the inequalities. The above objective chooses the one which has the smallest total number of bonds. It might be more appropriate instead to try and get the difference between the asset and the liability portfolio durations even closer, i.e. instead of getting just 
the durations to match $D_{A}=D_{L}$, have the errors in the durations to agree as well, so $D_{A}=D_{L}$ and $E_{A}\left(D_{A}\right)=E_{L}\left(D_{L}\right)$. If the liability occurs at only one time point then $E_{L}\left(D_{L}\right)$ is always zero and so $E_{A}\left(D_{A}\right)$ should be as close to zero as possible. Then instead of taking the objective function to be to minimize $\sum_{j} x_{j}$, one seeks to minimize $\sum_{j} x_{j}+\sum_{j} E_{j}\left(D_{L}\right) x_{j}$ subject to one equality constraint $V_{A}=V_{L}$ and a set of inequality constraints (19).

The other strategies used in immunization include matching partial (or key rate) durations of asset with those of liability. If no short selling of bonds is allowed, then, unfortunately, quite often there are no feasible solutions satisfying all equality constraints. One has to remove the restriction of short selling of bonds in partial or key rate duration matched strategies.

Partial duration matched strategy. The fourth approach is to match partial durations of the bond portfolio with those of the liability. Assume the model (5) is used to describe spot rate term structure. One can compute partial durations with respect to short rate, long rate, and short rate slope for each bond by (9) and then combine them together to get partial durations for the bond portfolio as in the case of Macaulay duration (16). The optimal portfolio is selected by minimizing the total number of bonds bought or sold $\sum_{j}\left|x_{j}\right|$ subject to four equality constraints (one value matched constraint and three partial duration matched constraints). Note that the above optimization problem is a linear programming problem by writing $x_{j}=x_{j}^{+}-x_{j}^{-}$with $x_{j}^{+}, x_{j}^{-} \geq 0$ and $\left|x_{j}\right|=x_{j}^{+}+x_{j}^{-}$.

Key rate duration matched strategy. The fifth approach is to match key rate durations of the bond portfolio with those of the liability. Assume a set of key rates determines changes of the term structure. Again one can compute key rate durations for each bond and then combine them together to get those for the bond portfolio. The optimal portfolio is selected by minimizing $\sum_{j}\left|x_{j}\right|$ subject to a set of equality constraints (one value matched constraint plus all key rate 
duration matched constraints).

\section{$5 \quad$ Horse Race Data and Result}

In this section performances of asset liability management with different duration strategies are compared using US Treasury STRIPS and Bonds. The risk free spot rate curves can be derived from US Treasury Bonds with bootstrapping technique or linear programming method. Since derived spot rates are very close to Treasury STRIPS rates (see Allen, Thomas, and Zheng [2000]), the latter are used to construct spot rate curves for simplicity. Exhibit 1 displays

Treasury STRIPS rates around February 15 from 1994 to 2001. Note that there were significant increases of interest rates in 1994 and 1999, and large decreases in 1995 and 2000. Six Treasury bonds are chosen each year to form a selection universe of the bond portfolio. All bonds are option free with face values of 100. Exhibit 2 lists the maturities and the coupons of these bonds. (For ease of calculation it is assumed that the coupons are paid annually).

A simple example is used to illustrate how the portfolios are set up, optimized, and rebalanced, for the different duration matching strategies. Suppose there is a two year liability from February 1999 to February 2001 with face value 100,000. A bond portfolio is set up in 1999 from six Treasury bonds in Exhibit 2. The prices of these bonds are computed by (3) where spot rates are 1999 Treasury STRIPS rates. The results are $V_{1}=103.52, V_{2}=112.94, V_{3}=103.72$, $V_{4}=104.13, V_{5}=102.13$, and $V_{6}=120.88$. The value of the liability is $V_{L}=91032.10$. Five portfolios are set up with different duration matched strategies. No short selling of bonds is allowed for Macaulay and approximate duration matched portfolios but this restriction is relaxed for the partial and key rate duration matched portfolios. For each strategy the corresponding durations for six bonds and the liability are first computed and then a linear programming 
problem is solved as discussed in detail in the previous section. The results are as follows:

Macaulay duration matched strategy. The yields of the bonds are computed by (1) and the Macaulay durations are then computed by (2). The results are $D_{1}=1.0, D_{2}=1.9, D_{3}=2.8$, $D_{4}=4.5, D_{5}=8.0$, and $D_{6}=13.4$. The Macaulay duration of the liability is $D_{L}=2.0$. The optimal portfolio is to buy 799 two year bonds and 6 twenty five year bonds.

Nearest integer approximate duration matched strategy. The approximate durations are computed by (14). The optimal portfolio is to buy 384 one year bonds and 424 twenty five year bonds.

Approximate duration matched strategy. The approximation errors are computed by (13) with $D_{L}=2$. The optimal portfolio is to buy 806 two year bonds.

Partial duration matched strategy. The four parameters in spot rate model (5) are first estimated by nonlinear regression using the 1999 US Treasury STRIPS rates. Partial durations to short rate, long rate, and short rate slope are then computed by (9). The optimal portfolio is to buy 686 two year bonds, 139 three year bonds, 1 twenty five year bond, and to short sell 9 ten year bonds.

Key rate duration matched strategy. The first, fifth, and twenty-fifth year spot rates are chosen as key rates and their durations are computed by (10) where $r_{0}(t)$ are the 1999 US Treasury STRIPS rates. The optimal portfolio is to buy 665 two year bonds, 187 three year bonds, and to short sell 33 five year bonds.

The portfolio is balanced once a year. After a year the values of these bonds are composed of two parts: the coupon payments and the prices of the bonds computed using the 2000 Treasury STRIPS rates. Adding two parts together to get $V_{1}=108.50, V_{2}=116.82, V_{3}=105.75$, $V_{4}=103.24, V_{5}=97.10$, and $V_{6}=112.87$. The values of the bond portfolios are then computed 
to see their performances against the value of the liability which is $94,020.31$. The results are that Macaulay duration gains 60.16, nearest integer approximate duration loses 4479.69, approximate duration gains 139.16, partial duration loses 8.31, and key rate duration gains 1.26. All bonds are sold or bought and gains or losses are brought forward to maturity of the liability (February 2001).

The same process is now repeated for the second of the two years, where there is a one year liability (from February 2000). All duration matched strategies produce the same optimal solution in the second year of buying 928 one year bonds which hedge the liability perfectly, i.e., the gains/losses at the maturity of the liability (February 2001) are zero, except the nearest integer approximate duration matched strategy that produces an optimal solution of buying 402 one year bonds and 467 two year bonds which gains 1135.82 at the maturity of the liability. Adding all gains/losses together to conclude that Macaulay duration gains 63.99, nearest integer approximate duration loses 3628.78, approximate duration gains 148.01, partial duration loses 8.84, and key rate duration gains 1.34 .

A set of horse races are carried out: The liabilities all have face values of 100,000 but with different length of durations in the range of two to seven years. All portfolios are set up and analyzed using observed market US STRIPS and Treasury bond data. The gains/losses of portfolios with different duration strategies are listed in Exhibit 3.

The approximate duration matched strategy does well in all tests: it has the largest average gain of 343.00 , it has the smallest maximum loss of 125.00 , and it has the largest maximum gain of 1089.16. If short selling of bonds is not allowed, it is also the safest method (with the smallest standard deviation of 434.54). Key rate duration matched strategy is a suitable choice for immunization since it has the smallest standard deviation, small maximum loss, and 
near-zero average gain/loss. Partial duration matched strategy is similar to but not as good as key rate duration matched strategy in every aspect, therefore it may only be used as a second choice in immunization. The biggest disadvantage of the key rate and the partial duration matched strategies is that short selling of bonds must be allowed to ensure feasible solutions and in practice this may not be possible. Macaulay duration matched strategy has reasonable performance in immunization, but is inferior to the approximate duration matched strategy. The nearest integer approximate duration matched strategy is the worst performer in all tests. It can make huge profits or suffer severe losses depending on how term structures of interest rates change. It is therefore not a suitable strategy in immunization.

\section{Conclusions}

In this paper the approximate duration is proposed to measure the sensitivity of bond prices to changes of interest rates and to use it in bond portfolio immunization. The approximate duration matched strategy is compared with Macaulay and other commonly used duration matched strategies using the US Treasury STRIPS and Bond data. The horse race result shows that approximate duration matched strategy is a possible contender for an asset liability management strategy: it does not assume any particular structures or patterns of changes of interest rates (in contrast to Macaulay duration), it does not need short selling of bonds (in contrast to key rate and partial durations), it is easy to find the optimal portfolio with linear programming, and it is robust to changes of interest rates. 


\section{$7 \quad$ References}

Allen, D. E., L. C. Thomas, and H. Zheng. "Stripping Coupons with Linear Programming." Journal of Fixed Income, September 2000, pp. 80-87.

Bierwag, G. O. Duration Analysis: Managing Interest Rate Risk. Cambridge, MA: Ballinger, 1987.

Bierwag, G. O., C. J. Corrado, and G. G. Kaufman. "Computing Durations for Bond Portfolios." Journal Of Portfolio Management, Fall 1990, pp. 51-55.

Birge, J. R., and F. Louveaux. Introduction to Stochastic Programming. New York: SpringerVerlag, 1997.

Chambers, D. R., W. T. Carleton, and R. W. McEnally. "Immunizing Default-free Bond Portfolios with a Duration Vector." Journal of Financial and Quantitative Analysis, vol. 23, 1988, pp. 89-104.

Cooper, I. A. "Asset Values, Interest Rate Changes and Duration." Journal of Financial and Quantitative Analysis, vol. 14, 1977, pp. 343-349.

Dahl, A. "A Flexible Approach to Interest Rate Risk Management," in Financial Optimization, ed. S.A. Zenios. Cambridge University Press, 1993, pp. 189-209.

Haugen, R. A. Modern Investment Theory. London: Prentice Hall International, 1997.

Ho, T. "Key Rate Durations: Measures of Interest Rate Risks." Journal of Fixed Income, September 1992, pp. 29-44. 
Exhibit 1. US Treasury STRIPS rates, 1994-2001.

\begin{tabular}{|c|c|c|c|c|c|c|c|c|}
\hline Year & 1994 & 1995 & 1996 & 1997 & 1998 & 1999 & 2000 & 2001 \\
\hline 1 & 3.91 & 6.72 & 4.70 & 5.51 & 5.41 & 4.81 & 6.36 & 4.33 \\
\hline 2 & 4.44 & 7.08 & 4.83 & 5.81 & 5.41 & 4.81 & 6.53 & 4.69 \\
\hline 3 & 4.85 & 7.33 & 4.92 & 5.93 & 5.42 & 4.89 & 6.58 & 4.77 \\
\hline 4 & 5.17 & 7.43 & 5.03 & 6.01 & 5.47 & 4.90 & 6.66 & 4.85 \\
\hline 5 & 5.44 & 7.40 & 5.14 & 6.09 & 5.50 & 4.93 & 6.74 & 4.91 \\
\hline 6 & 5.61 & 7.44 & 5.32 & 6.17 & 5.55 & 5.01 & 6.72 & 4.97 \\
\hline 7 & 5.79 & 7.51 & 5.47 & 6.26 & 5.60 & 5.05 & 6.75 & 5.07 \\
\hline 8 & 5.95 & 7.57 & 5.61 & 6.36 & 5.66 & 5.12 & 6.79 & 5.15 \\
\hline 9 & 6.09 & 7.61 & 5.75 & 6.43 & 5.72 & 5.18 & 6.80 & 5.20 \\
\hline 10 & 6.21 & 7.65 & 5.85 & 6.49 & 5.77 & 5.28 & 6.80 & 5.25 \\
\hline 11 & 6.34 & 7.69 & 5.94 & 6.55 & 5.83 & 5.39 & 6.80 & 5.33 \\
\hline 12 & 6.43 & 7.73 & 6.02 & 6.61 & 5.87 & 5.48 & 6.79 & 5.41 \\
\hline 13 & 6.53 & 7.76 & 6.09 & 6.65 & 5.91 & 5.55 & 6.78 & 5.49 \\
\hline 14 & 6.61 & 7.78 & 6.16 & 6.70 & 5.95 & 5.61 & 6.77 & 5.55 \\
\hline 15 & 6.69 & 7.79 & 6.22 & 6.74 & 5.98 & 5.66 & 6.76 & 5.62 \\
\hline 16 & 6.78 & 7.81 & 6.28 & 6.78 & 6.00 & 5.72 & 6.74 & 5.66 \\
\hline 17 & 6.84 & 7.83 & 6.33 & 6.81 & 6.01 & 5.76 & 6.70 & 5.70 \\
\hline 18 & 6.91 & 7.85 & 6.38 & 6.84 & 6.03 & 5.79 & 6.68 & 5.70 \\
\hline 19 & 6.93 & 7.85 & 6.41 & 6.86 & 6.05 & 5.79 & 6.67 & 5.73 \\
\hline 20 & 6.95 & 7.85 & 6.46 & 6.88 & 6.05 & 5.80 & 6.66 & 5.73 \\
\hline 21 & 6.97 & 7.85 & 6.48 & 6.90 & 6.06 & 5.79 & 6.65 & 5.75 \\
\hline 22 & 6.98 & 7.86 & 6.51 & 6.91 & 6.06 & 5.78 & 6.63 & 5.74 \\
\hline 23 & 6.99 & 7.86 & 6.52 & 6.92 & 6.07 & 5.76 & 6.57 & 5.73 \\
\hline 24 & 6.98 & 7.85 & 6.52 & 6.91 & 6.06 & 5.70 & 6.54 & 5.70 \\
\hline 25 & 6.97 & 7.83 & 6.52 & 6.90 & 6.06 & 5.64 & 6.47 & 5.69 \\
\hline
\end{tabular}

Source: The Wall Street Journal, New York Edition. 
Exhibit 2. US Treasury Bonds Used in Asset Portfolios, February 1994-2000.

\begin{tabular}{c|c|rrrrrrr} 
Bond & Maturity & 1994 & 1995 & 1996 & 1997 & 1998 & 1999 & 2000 \\
\hline 1 & 1 & $7 \frac{3}{4}$ & $7 \frac{7}{8}$ & $6 \frac{3}{4}$ & $7 \frac{1}{4}$ & $8 \frac{7}{8}$ & $8 \frac{1}{2}$ & $7 \frac{3}{4}$ \\
2 & 2 & $4 \frac{5}{8}$ & $4 \frac{3}{4}$ & $7 \frac{1}{4}$ & $8 \frac{7}{8}$ & $8 \frac{1}{2}$ & $11 \frac{3}{4}$ & $14 \frac{1}{4}$ \\
3 & 3 & $6 \frac{3}{4}$ & $8 \frac{1}{8}$ & $5 \frac{1}{2}$ & $8 \frac{1}{2}$ & $7 \frac{3}{4}$ & $6 \frac{1}{4}$ & $5 \frac{1}{2}$ \\
4 & 5 & $8 \frac{7}{8}$ & $8 \frac{1}{2}$ & $11 \frac{3}{4}$ & $14 \frac{1}{4}$ & $10 \frac{3}{4}$ & $5 \frac{7}{8}$ & $7 \frac{1}{2}$ \\
5 & 10 & $5 \frac{7}{8}$ & $7 \frac{1}{2}$ & $9 \frac{3}{8}$ & $6 \frac{1}{4}$ & $5 \frac{1}{2}$ & $5 \frac{1}{2}$ & $5 \frac{1}{2}$ \\
6 & 25 & $8 \frac{7}{8}$ & $8 \frac{1}{2}$ & $7 \frac{3}{8}$ & $7 \frac{7}{8}$ & $7 \frac{1}{8}$ & $7 \frac{1}{8}$ & $7 \frac{5}{8}$
\end{tabular}

Source: The Wall Street Journal, New York Edition.

Exhibit 3. Gains/Losses of Duration Matched Strategies in Immunization

\begin{tabular}{l|rrrrr} 
Liability/Strategy & Macaulay & NI Approx & Approx & Partial & Key Rate \\
\hline Two Year: 1999-2001 & 63.99 & -3628.78 & 148.01 & -8.84 & 1.34 \\
Three Year: 1998-2001 & 79.94 & -3192.03 & 24.81 & -22.54 & 3.74 \\
Four Year: 1997-2001 & 93.31 & -3341.83 & -125.00 & -76.43 & 116.87 \\
Five Year: 1996-2001 & 529.74 & -2729.30 & 487.54 & -0.24 & 61.67 \\
Six Year: 1995-2001 & -803.86 & 966.40 & 433.50 & -224.01 & -135.17 \\
Seven Year: 1994-2001 & 343.92 & 119.50 & 1089.16 & -479.75 & -264.01 \\
\hline Average Gain/Loss & 51.17 & -1967.67 & 343.00 & -135.31 & -35.93 \\
Standard Deviation & 457.95 & 1984.53 & 434.54 & 188.13 & 139.79 \\
Maximum Loss & -803.86 & -3628.78 & -125.00 & -479.80 & -264.01 \\
Maximum Gain & 529.74 & 966.40 & 1089.16 & -0.24 & 116.87
\end{tabular}

